\section{La percepción de los costos de producción del banano orgánico en el Cantón Machala, Ecuador}

\author{
The perception of the production costs of the organic banan \\ in Cantón Machala, Ecuador
}

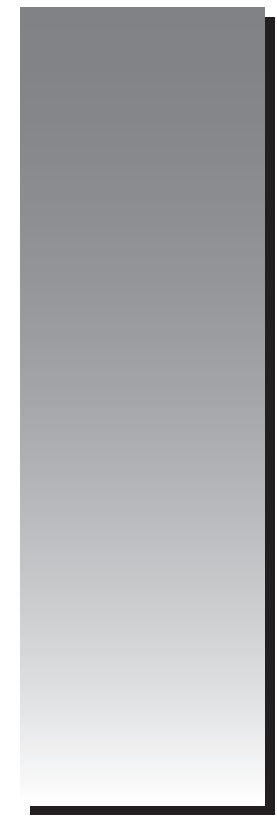

Félix Armando Rivera León

friveral@unmsm.edu.pe

Lenny Beatriz Capa Benítez

lenyca27@hotmail.com

Robinson Miguel Benítez ambiente.

\begin{abstract}
The present study is aimed at analyzing the perception of indirect manufacturing costs as one of the relevant nodes in organic banana production in Canton Machala companies in Ecuador. Different theoretical methods such as analysis and synthesis and modeling are used to achieve the objective. As an empirical method, a survey was applied to a sample of 66 organic banana companies, which was processed through the statistical package SPSS 23.0. The main results are that in organic banana companies the perception of costs is examined from three key moments of the production process: before, during, after production, and at the same time provides an analysis approach that improves the aggregation management of the costs of production in an environmental environment.
\end{abstract}

Keywords: Costs; organic products; production; bananas; environment. \section{Narváez \\ robinsonbenitez@hotmail.com \\ Universidad Nacional Mayor de San Marcos, Facultad de Ciencias Administrativas}

(c) Los autores. Este artículo es publicado por Gestión en el Tercer Milenio de la Facultad de Ciencias Administrativas de la Universidad Nacional Mayor de San Marcos. Este es un artículo de acceso abierto, distribuido bajo los términos de la licencia Creative Commons Atribucion - No Comercia_Compartir Igual 4.0 Internacional. (http://creativecommons.org/licenses/by-nc-sa/4.0/) que permite el uso no comercial, distribución y reproducción en cualquier medio, siempre que la obra original sea debidamente citada. 


\section{INTRODUCCIÓN}

La agricultura orgánica es una estrategia de desarrollo que trata de cambiar limitaciones de la producción convencional. Además de constituir una tecnología de producción, representa una estrategia de desarrollo basada en el adecuado manejo del suelo, el incentivo al uso de insumos locales, así como, aportando un mayor valor agregado y una cadena de comercialización más justa. (FAO, 2003)

El incremento paulatino de la demanda de productos orgánicos, básicamente en países desarrollados desde finales de los 80', provocó la proliferación de las prácticas de la agricultura orgánica en muchos países por lo que hoy es considerada como una oportunidad, no solo porque el mercado demanda cada vez más productos libres de contaminantes, sino, que el aumento de la población mundial demanda más alimentos y si ellos tienen un menor impacto ambiental favorecen el desarrollo sostenible. (Martínez, Bello, \& Castellanos, 2012)

En correspondencia, la producción de productos orgánicos se ha extendido a muchos países de Europa, Asia y América Latina, en esta última, Ecuador se destaca por su participación con varios productos como es el cacao, café, caña y el banano.

La producción de banano orgánico, desde hace varios años, ha alcanzado un auge en el desarrollo socioeconómico del Ecuador, no solo por su aporte al Producto Interno Bruto (PIB), el cual está en aumento cada año. Constituye una importante fuente de empleo y estos elementos se manifiestan con fuerza específicamente en el Cantón Machala en la Provincia de El Oro.

Sin embargo, una de las interrogantes más frecuentes en el mercado del banano orgánico es por qué los precios de este producto tienden a ser más altos que el del banano convencional.

La FAO (Organización de las Naciones Unidas para la Agricultura y la Alimentación), alega varias razones entre ellas los costos de producción son más elevados porque requieren mayor cantidad fuerza de trabajo por unidad de producción, porque diversidad de las empresas no posibilita obtener economías de escala, en la post cosecha también se incrementan los costos, lo mismo que en la cadena de comercialización y distribución, que resulta en cierta forma ineficiente y los costos son más elevados por tratarse de volúmenes relativamente pequeños. (FAO, 2016)

Estos argumentos generan la motivación de la presente investigación, relacionada con los costos del banano orgánico, que se presenta como una herramienta eficaz a través de la cual se puede medir el comportamiento de la actividad económica de la empresa, proporcionando información necesaria para la toma de decisiones.

Durante el proceso de producción del banano orgánico, las empresas realizan operaciones encaminadas a la protección del medio ambiente, sin dejar de prestar atención a la obtención de altos niveles de rentabilidad; sin embargo, aún no tienen resultados o falencias relacionadas con los costos indirectos asociados con el cuidado del medio ambiente.

En correspondencia, es fundamental estudiar la dinámica de los costos del banano orgánico antes, durante y después de la producción; como un elemento fundamental para mejorar constantemente su competitividad en el mercado.

La problemática de este estudio está en cómo se manifiesta percepción de los costos indirectos de fabricación, como uno de los nudos relevantes, en la producción del banano orgánico en las empresas del Cantón Machala en Ecuador.

Atendiendo a estos elementos, el objetivo de la presente investigación es analizar la percepción de los costos indirectos de fabricación, como uno de los nudos relevantes, en la producción del banano orgánico en las empresas del Cantón Machala en Ecuador.

\section{CONSIDERACIONES RELEVANTES.-}

Según Hargadon \& Múnera (2007) en las empresas industriales se distinguen tres funciones básicas: producción, ventas y administración y que para desarrollarlas tiene que efectuar ciertos desembolsos por pago de salarios, arrendamientos, servicios públicos, 
materiales, etc. Estas erogaciones reciben el nombre de costos de producción, gastos de administración y gastos de ventas, según la función a que pertenezcan.

En tal sentido, los costos representan erogaciones y cargos asociados, clara y directamente, con la adquisición o la producción de los bienes o la prestación de los servicios, de los cuales un ente económico obtendrá sus ingresos. (Sinisterra, 2007)

Para Horngren, Madhav, \& Srikant, (2012), es un sacrificio de recursos que se asigna para lograr un objetivo específico.

En el estudio de los costos es importante distinguir si estos tienen relación directa o indirecta con un objeto de costos específico. Al respecto se presentan los llamados costos directos e indirectos de producción.

Los costos directos están relacionados con el objeto de costos en particular y pueden cargarse a dicho objeto desde el punto de vista económico. Sin embargo, los costos indirectos, aunque igualmente se relacionan con un objeto de costo en particular, no pueden atribuírsele desde el punto de vista económico, por lo que representa un desafío para las administraciones su asignación ya que no se identifican con un solo objeto de costo, sino con varios de ellos. (Horngren, Madhav, \& Srikant, 2012)

Los costos indirectos de fabricación (CIF), se agrupan en las categorías de materiales indirectos, mano de obra indirecta y otros gastos indirectos de fabricación. De lo anterior se infiere que estudiar los costos de producción en una empresa representa disponer de información para para medir la eficiencia de la producción, proporcionar información para la planificación de sus distintas operaciones porque, para tomar decisiones a mediano y largo plazo, la gerencia necesita comprender en principio los costos antes, durante y posterior a la producción.

\section{MÉTODO:}

El tipo de estudio es observacional analítico transversal, el cual se desarrolla en el Cantón de Machala de la Provincia de El Oro, en las empresas productoras de banano orgánico.
El total de empresas objeto de estudio lo constituyeron 66; es decir, el universo que cumplen con la producción de este producto de forma orgánica.

Para la recogida de la información se utiliza el método de la encuesta, la cual fue validada en sus seis dimensiones:

\section{Valides interna:}

- Validación de contenido, que es la creación del instrumento, tuvo en cuenta sus tres momentos: aproximación a la población; juicio de expertos y validez racional.

- Para la confiabilidad. Para esta dimensión, se utilizó el Alfa de Cronbach. Este estadígrafo muestra que la proporción de la varianza en los resultados, es verdadera. En este estudio se obtuvo 0,842. (Gil, 2015)

- Validez de constructo. Para su determinación se utilizó el "análisis factorial", que se complementó con el gráfico resumen de los factores.

- Validez externa:

- La validez de criterio se contrasta con el patrón de oro a través del grado de concordancia de Kendall. En esta investigación el valor del estadígrafo resultante fue de 0,86. (Gil, 2015)

- La estabilidad. Para poder medir esta dimensión se tomaron dos muestras como pilotos, resultando el valor de $85 \%$.

- El rendimiento fue determinado a través de su punto de corte utilizando la curva de ROC.

Para el análisis de los datos se utilizó el paquete estadístico SPSS versión 23.0 en castellano, donde el estadístico calculado fue la mediana debido a la medida de las variables que ¿está en una? Escala ordinal. Se utilizó los gráficos de cajas y bigotes con la finalidad de complementar la interpretación que se muestra en el estudio. 


\section{RESULTADOS Y DISCUSIONES.-}

\section{Hallazgo:}

Como se muestra en la figura 1, existen dos ítems que están cercanos al cinco, que es la cualidad mayor, lo que expresa que la gestión y proyección del cultivo orgánico obedece a una planificación y que dentro del ciclo productivo se consideran aspectos relacionados con el medioambiente antes de la producir.

\section{Discusión:}

Con resultados contrarios, aparece que la productora no cuenta con metas de ambientales medibles y verificables en un plazo de tiempo definido ni con metas de proyección social certificables en un plazo de tiempo definido.

\section{Hallazgo:}

En la figura 2, se evidencia que las percepciones durante la producción, a esto los encuestados le conceden una alta valoración a un número mayor de ítems como son los casos de que la productora contrata servicios de consultoría y capacitación en costos de producción y en aspectos medioambientales. De igual manera poseen certificaciones para su operación y autonomía para mejorar consideraciones no programadas, durante la producción.

El ítem menos favorecido resultó que al producir no considera la pertinencia de costos de producción.

\section{Discusión:}

La capacitación constituye un elemento fundamental dentro de los costos indirectos, que debe ser considerado en la producción del banano orgánico; de manera que cada productor adquiera los conocimientos y destrezas necesarias para lograr una producción los volúmenes de producción planificados.

\section{Hallazgo:}

En lo que respecta a la percepción posterior a la producción, como se muestra en la figura 3, fueron valorados tres ítems con alta puntuación que significa que la productora ha contratado servicios de consultoría y capacitación en aspectos medioambientales; que el proceso de toma de decisiones en la empresa, a menudo, tiene que ver con los costos de producción y que la planta productora posee autonomía para corregir consideraciones posteriores a la producción.

\section{Discusión:}

Con una valoración baja, se encuentran que el personal y la información que ellos generan es clave para la adopción de nuevas decisiones. Que la productora no cuenta con metas ambientales medibles y verificables en un plazo de tiempo definido ni con metas de proyección social medibles y verificables en un plazo de tiempo definido.

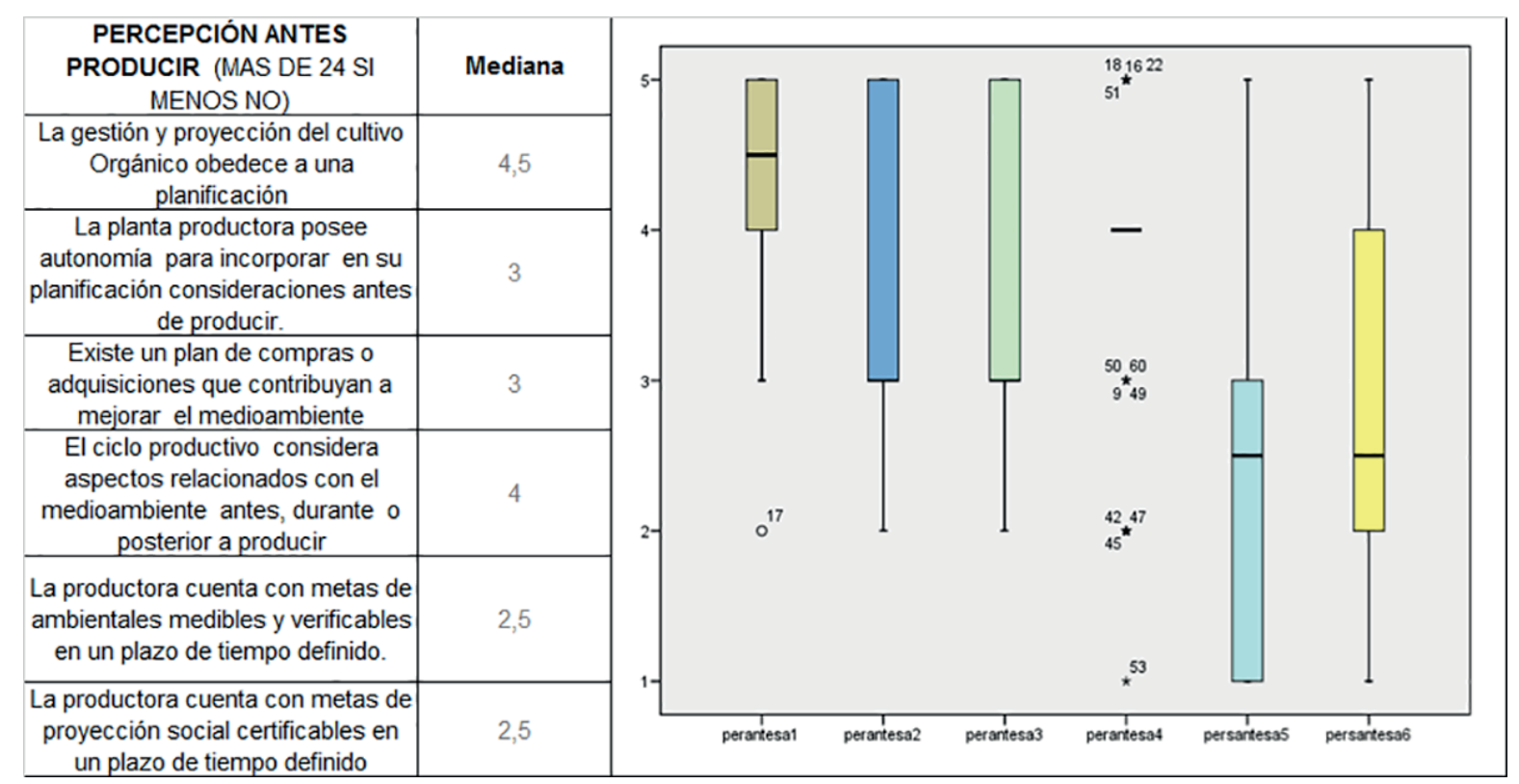

Figura1. Planificación y gestión de costos medioambientales. Percepción antes de la producción. Machala 2016. Fuente: Encuesta 


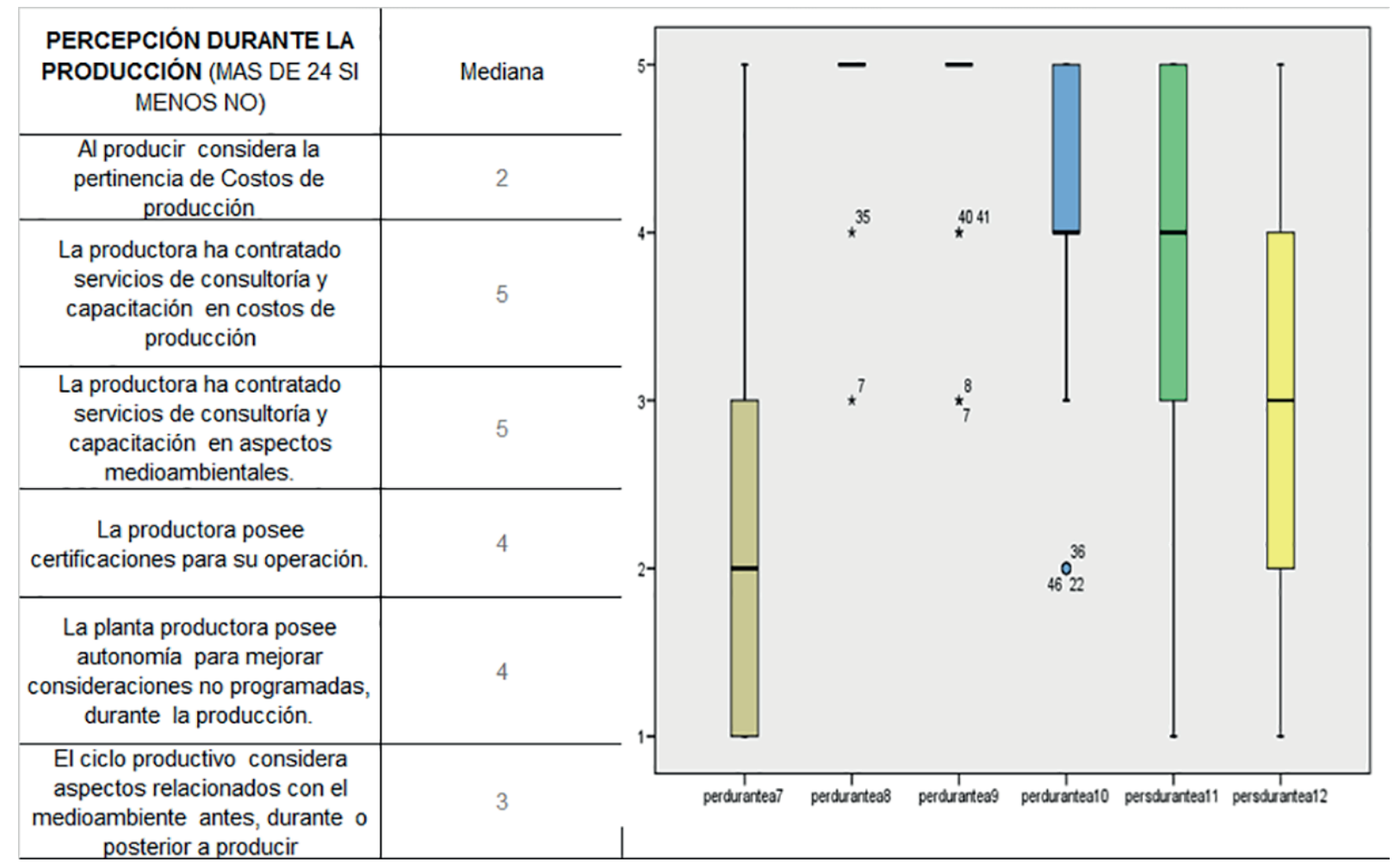

Figura 2. Planificación y gestión de costos medioambientales. Percepción durante la producción. Machala 2016. Fuente: Encuesta

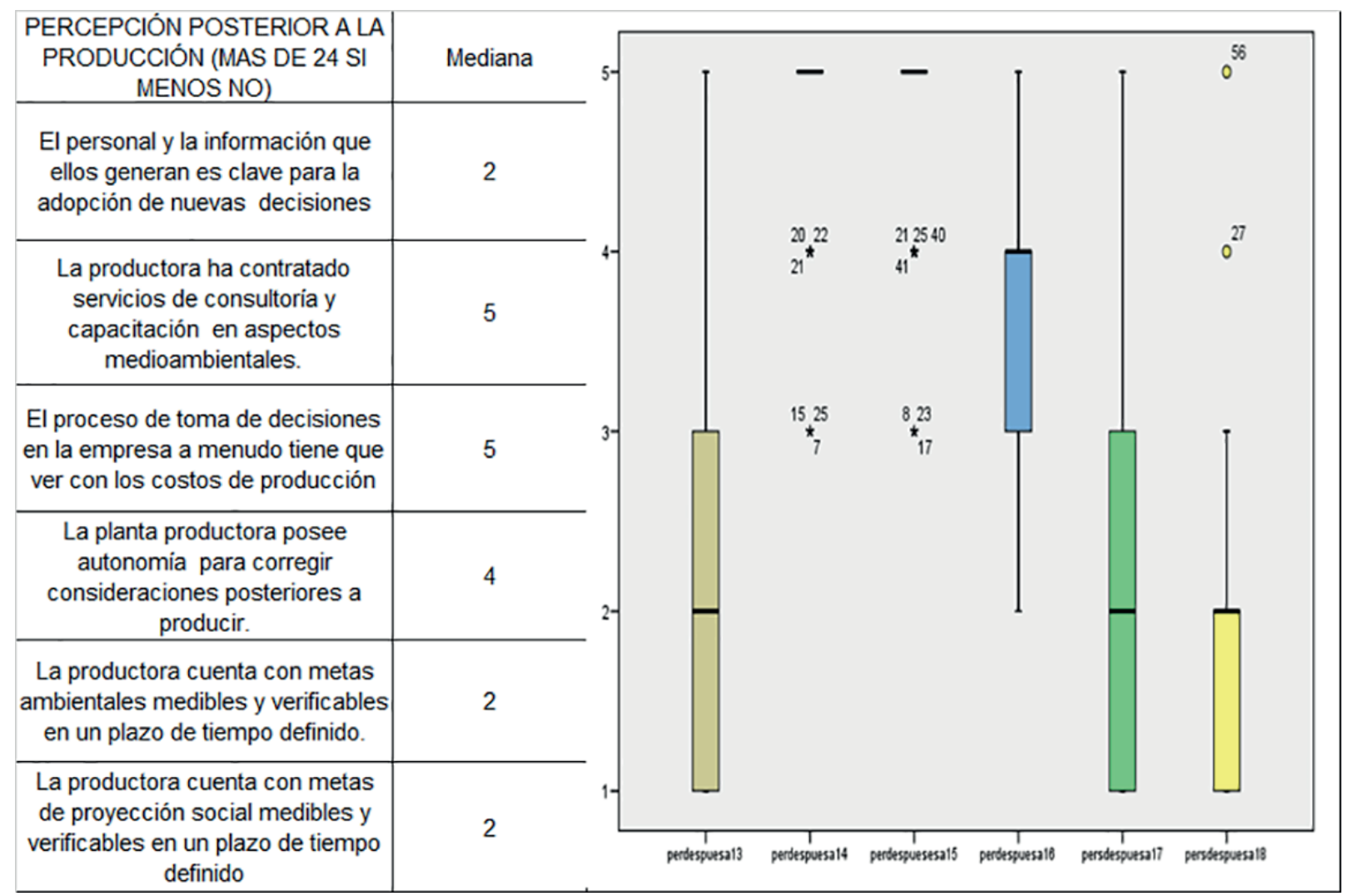

Figura 3. Planificación y gestión de costos medioambientales. Percepción posterior a la producción. Machala 2016. Fuente: Encuesta 
En el ítem relacionado con la planificación, estos resultados coinciden con los declarados en la literatura donde se expresa que uno de los problemas más importantes que se ha observado en este tipo de organización es la falta de planeación, expectativas claras y realistas; asociadas a las metas ambientales a la que aspira la empresa. (FAO 0. d., 2003), (Osuna, Vázquez, Preciado, \& Fortiz, 2015);

Los resultados, expresados en este análisis, demuestran que las empresas productoras de banano orgánico en el Cantón Machala carecen de una percepción sistémica al momento de planificar y gestionar los costos indirectos, antes, durante y después de la producción; destacándose que no cuenta con metas de ambientales medibles y verificables en un plazo de tiempo, no consideran la pertinencia de costo de producción ni cuentan con metas concretas referidas al cuidado y protección del medio ambiente.

\section{CONCLUSIÓN:}

Se ha evidenciado, en el estudio, que la percepción de los costos indirectos de fabricación, como uno de los nudos relevantes en la producción del banano orgánico en empresas del Cantón Machala en Ecuador, antes, durante y después; muestran falencias que constituyen un obstáculo para la correcta planificación y gestión del costo indirecto en la producción del banano orgánico, con una perspectiva tanto a largo como mediano plazo.

Carecer de estos elementos, limita a las empresas disponer de información necesaria para medir la eficiencia de la producción del banano orgánico de forma correcta y, además, realizar análisis prospectivos que logre un desarrollo sostenible y competitivo en este mercado, cada vez más creciente y exigente.

\section{REFERENCIAS BIBLIOGRÁFICAS:}

Coeficiente de Kendall-Ecu Red.-https:/www. ecured.cu/Coeficiente Kendall.

Cronbach L (1951) Coeffient Alpha and the Internal Structure of Test. Psycometrika (setiembre) pp 297-334.FAO. (5 de Agsto de 2016).

Curva ROC - Wikipedia, la enciclopedialibre:https:// es.wikipedia.org/wiki/Curva_ROC

OrganicAgriculture. Obtenido de http://www.fao. org/organicag/oa-faq/oa-faq5/es/

FAO, O. d. (2003). Agricultura Orgánica: una herramienta para el desarrollo rural sostenible y la reducción de la pobreza. Costa Rica: Memoria del Taller.

Gil, J. (2015). Estadística e Informática (SPSS) en la Investigación descriptiva en inferencial. Madrid: Educación Nacional de Educación a Distancia.

Hargadon, B., \&Múnera, A. (2007). Contabilidad de Costos. Bogotá: Norma S.A.

Horngren, C., Madhav, R., \&Srikant, D. (2012). Contabilidad de Costos un enfoque gerencial. México: Pearson Educación.

Martínez, L., Bello, P., \& Castellanos, O. (2012). Sostenibilidad y desarrollos: el valor agregado de la agricultura orgánica. Bogotá: Programa de Investigación en Gestión, Productividad y Competitividad - BioGestión.

Osuna, P., Vázquez, C., Preciado, P., \&Fortiz, M. (2015). Agricultura orgánica: octava parte. México: Red Internacional de Agricultura Orgánica.

Padilla, G. (2015). Contenidos Especializados en la Enseñanza Superio. ACCI.

Sinisterra, G. (2007). Contabilidad de Costos. Bogotá: McGraw- Hill . 